\title{
Implicancias de los riesgos laborales percibidos por las enfermeras en el valor concedido al cuidado y al cuidador en el Servicio de Medicina Interna del Hospital Nacional Guillermo Almenara Irigoyen, Lima, 2016
}

\author{
Implications of labour risks perceived by nurses in value granted to care and the caretaker in the \\ Internal Medicine Service Hospital Nacional Guillermo Almenara Irigoyen, Lima, 2016
}

Irene Zapata Silva ${ }^{*}$

RESUMEN

\begin{abstract}
Objetivo: describir y analizar la percepción de los riesgos laborales de las enfermeras en el proceso de cuidado y sus implicancias. Metodología: fue un estudio cualitativo, mediante etnometodología, realizado en una muestra de once enfermeras del servicio de medicina interna definida por criterio de saturación. Los instrumentos fueron entrevistas semiestructuradas y observación participante. Resultados: se obtuvo tres etnocategorías, analizas por análisis temático: (1) Riesgo de contraer enfermedades infecciosas y punciones accidentales; sufrir lesiones por sobrecarga; implicación emocional y estrés; sufrir agresión y verse inmerso en demandas legales. (2) Las implicancias la enfermera fueron déficit en la salud física y emocional y (3) Para la Institución es urgente superar los limitantes: mobiliario y equipo obsoleto, el valor del cuidado y de quien cuida, por una cultura preventiva. Conclusiones: La percepción de los riesgos laborales y sus implicancias, se revela en multiplicidad de situaciones riesgosas, siendo emergentes el riesgo de ser agredido y verse inmerso en demandas legales por la nueva configuración del usuario-ciudadano. Compete a las autoridades gestionar, la creación de condiciones saludables para la realización del cuidado y el cuidado de la enfermera; se precisa mayor postura crítica y actitud transformadora de las condiciones laborales existentes por parte de las enfermeras, acorde a la Legislación existente en materia de Seguridad y Salud en el trabajo; estrategia para mitigar y resolver una de las mayores disonancias: el estrés laboral. Las enfermeras reconocen que el cuidado es "trabajo necesario para los seres humanos, implicando cuotas de desgaste muy altas”.
\end{abstract}

Palabras clave: Enfermería, Hospitalización, Riesgos laborales, cuidador

\begin{abstract}
Objective: To describe and analyze the perception of occupational hazards of nurses in the care process and its implications. Methods: a qualitative study was by etnometodología conducted in a sample of eleven nurses of internal medicine defined by saturation criterion. The instruments were semi-structured interviews and participant observation. Results: Three ethnocategories was obtained, you look for thematic analysis: (1) Risk of infectious diseases and accidental punctures; overuse injuries; emotional involvement and stress; suffer aggression and being immersed in lawsuits. (2) The implications nurse were deficits in physical and emotional health and (3) the institution is urgent to overcome the limitations: furniture and obsolete equipment, the value of care and who cares for a preventive culture. Conclusions: the perception of occupational risks and their implications, revealed in multiple risky situations, being emerging risk being attacked and being immersed in lawsuits by the new configuration of user-citizen. Authorities must manage, creating healthy conditions for the realization of care and nursing care; more critical stance and attitude of transforming existing labor conditions by nurses is required, according to the existing legislation on health and safety at work; strategy to mitigate and solve one of the greatest discords: work stress. Nurses recognize that care is "work necessary for humans, implying very high wear quotas".
\end{abstract}

Keywords: Nursing, Hospital, Occupational risks, caregiver

${ }^{1}$ Doctora en Enfermería. Miembro del equipo de Gestión de la Calidad. Hospital Guillermo Almenara Irigoyen, Lima, Perú. 


\section{INTRODUCCIÓN}

La recuperación de la salud de las personas ha evolucionado considerablemente, requiriendo diversos niveles de atención, tecnología y potencial humano de diversa capacidad resolutiva, factores estrechamente ligados al proceso de cuidado que realiza la enfermera como el modo de ser y estar con la persona cuidada (Waldow 1998). En este proceso se llevan a cabo innumerables actividades que insumen conocimientos, habilidades, técnicas e instrumentos a fin de satisfacer necesidades específicas del paciente según el grado de compromiso en su déficit de salud; actividades que implican no solo interrelación permanente sino también carga mental y emocional por la continuidad de las acciones, el sentido prioritario y el impacto personal que caracteriza la respuesta humana de quien enfrenta la enfermedad (Creus 2013).

A nivel mundial trabajan en salud 59,2 millones de personas; en la Región de las Américas son cerca de 21 millones, siendo un hecho que cada año mueren dos millones de trabajadores por accidentes, enfermedades o lesiones relacionadas con el trabajo realizado. Asimismo se sabe que la tasa global de trastornos depresivos asociados a los riesgos ocupacionales es del 8\%. En el Perú, del total de enfermeras que trabaja en el Sector estimado en 24,279 (2009), el 57\% se desempeña en servicios hospitalarios públicos y privados (MINSA 2013).

En el escenario del cuidado, el riesgo laboral es un hecho permanente en la multidimensionalidad de las intervenciones profesionales. No obstante las competencias adquiridas durante la formación básica permiten enfrentar la vulnerabilidad integral que como ser humano alcanza a la enfermera, a fin de evitar que diferentes agentes de tipo físico, químico, radiológico, ambiental, entre otros, actúen ocasionando daños a su salud (Cardona 2010). Sin embargo el carácter constante y prolongado de la exposición laboral ha sido reconocido internacionalmente no sólo respecto al riesgo biológico (Santos y Mauro 2009), sino principalmente emocional por la naturaleza de las responsabilidades asumidas como el responder por la vida de aquéllos cuyo cuidado se nos confía, siendo denominada enfermería, una de las profesiones más estresantes en el sector salud (Murofuse, Abranches \& Napoleao 2005 y Meneguini 2011).

La experiencia laboral en áreas clínicas, la supervisión y la docencia, condujo a la observación de la exposición de la enfermera a los riesgos laborales como un hecho constante, planteando la interrogante ¿Cuál es la percepción de las enfermeras acerca de los riesgos laborales en el proceso de cuidados y las implicancias que conlleva? Desde el Paradigma hermenéutico o interpretativo, el conocimiento de la percepción de las enfermeras sobre los riesgos laborales y sus implicancias en el valor del cuidado y de quien cuida, constituyen la base para la gestión de la cultura de seguridad y el incremento de la adherencia a las medidas preventivas desde el significado que las profesionales construyen en su cotidianidad.

\section{MATERIAL Y MÉTODOS}

El escenario de estudio fue el servicio de medicina interna del 3B Este y Oeste, donde se brinda cuidados correspondientes a la categoría del hospital nacional Guillermo Almenara nivel III-2, entre los meses de mayo a julio 2015; el enfoque fue cualitativo de tipo etnometodológico según Garfinkeld (1960 en Firth 2010), quien propició el conocimiento del sentido que conceden las personas a sus acciones cotidianas, las reglas que construyen y las decisiones que adoptan; se sustentó en el referencial sociológico de Beck, Slovic y Luhmann, permitiendo la comprensión de la percepción de las enfermeras sobre los riesgos laborales a que están expuestas en el proceso de cuidado. Once enfermeras participaron en el estudio siendo definida la muestra por criterio de saturación; El tiempo de servicios fue superior a 6 años, siendo la condición laboral sin diferencia entre nombrados y contratados. Los instrumentos fueron la entrevista semiestructurada y la observación participante con registro en un diario de campo, en horarios comprendidos entre las 9am a 10.30am y de 2 a 3.30pm, de lunes a viernes. La información proporcionada fue grabada con el consentimiento de la participante. Los testimonios obtenidos fueron ordenados previo proceso de transliteración en tablas de doble entrada por temas; inicialmente en el lenguaje propio de los informantes o EMIC para luego mediante lecturas sucesivas y reflexión a la luz del referencial teórico, realizar la interpretación en el lenguaje ETIC o del investigador. Las categorías fueron analizadas mediante análisis temático de Bardin. El informe preliminar de resultados fue socializado con las enfermeras informantes a fin de validar la fidelidad de su contenido. A quienes aceptaron participar en el estudio se les solicitó firmar el formulario de consentimiento informado. 
Implicancias de los riesgos laborales percibidos por las enfermeras en el valor concedido al cuidado y al cuidador en el Servicio de Medicina Interna del Hospital Nacional Guillermo Almenara Irigoyen, Lima, 2016

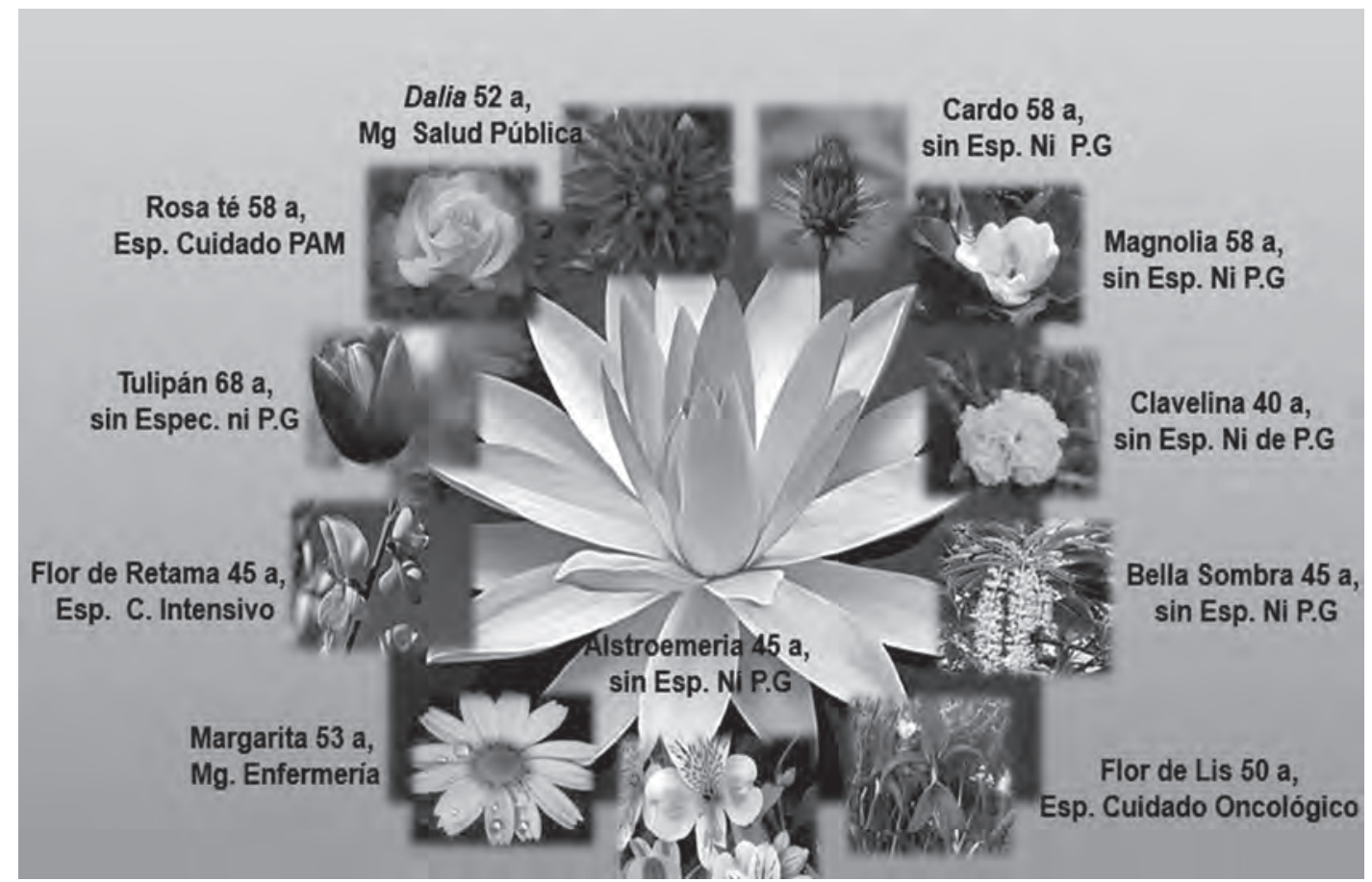

\section{RESULTADOS}

\section{Riesgos laborales en el proceso del cuidado cotidiano}

Esta categoría temática corresponde a la percepción en amplitud de los riesgos que enfrenta la enfermera en el proceso de cuidado, realizada mediante observación participante y entrevista semiestructurada, se evidencia una dinámica intensa en la multidimensionalidad de intervenciones a cargo de la enfermera: el cuidado directo, la gestión y la docencia; los riesgos emergentes se evidenciaron en varias subcategorías:

Subcategoría: contraer enfermedades infectocontagiosas y punciones accidentales,

(...) "Me tocó sufrir serias infecciones respiratorias después de las guardias, ningún antibiótico me daba resultados" (Clavelina).

(...) "Una de las situaciones más riesgosas para mí, está el cuidar a pacientes agitados cuando me quedo sola, también cuando debo encontrar un acceso venoso y el paciente no lo permite, puede moverse bruscamente y con ello el riesgo de sufrir una puntura accidental” (Bella sombra).

Subcategoría: riesgo por sobrecarga, posturas y movimientos repetitivos. Percibidos así:
(...) "Lo que más me preocupa es el esfuerzo físico, casi siempre salimos con dolor en la espalda y esto es más serio cuando las camas están malogradas, Tenemos para movilizar en esta sala cerca de 18 pacientes y solo 5 de ellos colaboran en su movilización, me faltan todavía muchos años de trabajo"(Calla un momento) (Dalia).

(...) "Por el estado de gran dependencia del paciente, sustituimos lo que él podía haber hecho, entonces el esfuerzo físico es más intenso," (Rosa Té).

Subcategoría: riesgo emocional y estrés. Una de las áreas más reveladoras del proceso de cuidado:

(...) "Más afectada me siento en la parte emocional, por las exigencias diarias que dejen huellas; notas la irritabilidad," (Flor de Lis).

(...) “. Si tuviera que hacer un balance de mi salud en el trabajo, diría que el trabajo en enfermería nos cambia, no tenemos la misma energía cuando empezamos...” (Dalia)

Subcategoría: agresión en el proceso de cuidado

(...) “... Un paciente me dio una patada en la espalda, y al día siguiente no podía caminar, tenía un desgarro muscular”. (Magnolia)

(...) "Un riesgo es ser atacado por un paciente o su familiar, el paciente cuando se desorienta y 
la familia cuando no aceptan la muerte de un ser querido, culpan al hospital y agreden al personal que les informa sobre lo sucedido, porque ningún otro profesional permanece en el servicio”. (Clavelina)

El Plan global de Salud y Seguridad en el trabajo contempla la necesidad de trabajar estrategias de prevención y canalización en la expresión de sentimientos hostiles del usuario con capacidad de protección al trabajador.

Subcategoría: implicación en demandas legales.

(...) "No quiero ni pensar en desfilar a dar declaraciones porque un paciente en mi turno, se quitó la vida y ya hubo más de un intento” (Rosa Té).

(...) "Mientras estaba canalizando una vía en salas generales, vi pasar un paciente que de pronto intentaba saltar por la ventana desde el tercer piso, casi muero del susto; corrí y pedí ayuda para evitar que se lanzara a pesar que sentía que el peso del paciente me ganaba. ¡Qué horror! Tiré la cubeta y solo pensé el paciente no puede hacerme esto, después van a decir que lo descuidé y estaba atendiendo a otro paciente y en la misma sala por suerte” (Tulipán).

\section{Implicancias de los riesgos laborales en la salud de la enfermera}

Corresponde a la percepción de las enfermeras acerca del impacto de la exposición a los diferentes riesgos laborales en su estado de salud, extraídos principalmente en los diálogos espontáneos durante los períodos de observación participante. Están expresados en los siguientes relatos:

(...) "Los riesgos laborales significaron para mi accidente, me puncé con un catéter contaminado...mi vida cambió..." (Rosa Té).

(...) "Saber que me había contagiado fue lo peor, la realidad me ha hecho cambiar. Me afectó mucho en mis sentimientos, vida de pareja, social, y en lo físico, me sentí aislado y culpable por no haberme cuidado lo suficiente”. (Tulipán).

Los siguientes relatos aluden a las condiciones de trabajo en enfermería y su modalidad por turnos, también la continuidad y prolongación de estas condiciones:

(...) "Me afecta con los años el frío en las guardias nocturnas, siento que necesito estar en mi cama en las noches" (Tulipán).
(...) "No me siento igual que antes, el cuidado de enfermería comprende carga mental, estar alerta, trabajo físico, interacción, cambios de turno y trabajo nocturno con días de descanso alternados que a veces me hace perder la noción del tiempo "(Alstroemeria).

(...) "El fuerte olor de la lejía que usan para la limpieza de pisos y paredes, me ocasionó edema de glotis, es asma ligada al trabajo” (Clavelina).

\section{Urgencia de superar los limitantes por una cultura preventiva}

La percepción de las enfermeras evidencia abastecimiento de insumos de patrón irregular, no se garantiza la protección permanente del personal, en tres subcategorías relevantes para el cuidado:

Subcategoría: mobiliario obsoleto, equipamiento no acorde a las demandas del cuidado.

Ante las deficientes condiciones de trabajo, las enfermeras realizan ingentes esfuerzos para elevar su nivel de inmunidad y vencer la creciente vulnerabilidad en su labor; son expresiones muy reveladoras:

(...) "No hay espacio para colocar el mobiliario, si hubiera un sismo, pacientes y personal tropezarían con todo. El hospital está atrasado en la adquisición de equipo, mobiliario y planta física, tal como el paciente lo necesita”. (Cardo).

(...) "Es necesaria la reposición y adquisición de equipos más modernos, ¿Por qué tenemos que sufrir tanto junto con los técnicos de enfermería? cuando hay más demanda, tenemos que desplazar los aspiradores de un sitio a otro entre cables y extensiones múltiples, esto no solo es más gasto de tiempo sino peligro de enredarnos y caer aparatosamente...”. (Clavelina).

Subcategoría: cuidado del cuidador

La enfermera realiza el cuidado respetando la normatividad y aplicando los recursos existentes, sin embargo se manifiestan situaciones de sobrecarga que representan mayor inversión de energía y dedicación, teniendo como consecuencia esta percepción:

(...) "Falta una visión más realista y humana, de que no podemos seguir trabajando así, que el mobiliario y el equipo se desgastan y tienen que ser renovados, igualmente el número de personal es insuficiente"(Bella Sombra).

Subcategoría: valor real del cuidado y de quien cuida 
Implicancias de los riesgos laborales percibidos por las enfermeras en el valor concedido al cuidado y al cuidador en el Servicio de Medicina Interna del Hospital Nacional Guillermo Almenara Irigoyen, Lima, 2016

(...) "Les he contado mi experiencia para que aprendan, sabiendo que uno ni siquiera piensa que le puede suceder... en el momento menos pensado puede hacer la diferencia entre ser un trabajador sano o sufrir TBC pulmonar” (Tulipán).

(...) "Para la limpieza usan soluciones con sustancias químicas fuertes, inhalamos con daño a la vía respiratoria. Las condiciones en las que trabajamos no son buenas: hay trabajo de alta demanda, ambiente reducido, mala ventilación y comodidades mínimas" (Flor de Retama).

Las enfermeras perciben la existencia de condiciones riesgosas en el proceso de cuidado, su salud es comprometida seriamente y la disposición que muestran las autoridades revela desconocer las consecuencias de esta situación, a la par que indica desvaloración del cuidado y de la persona que brinda el cuidado.

\section{DISCUSIÓN}

La dinámica laboral desplegada por la enfermera en el proceso de cuidado se inserta entre avatares y satisfacciones por el bien que representa participar del esfuerzo por la restauración de la salud perdida. Como actores sociales en procesos altamente riesgosos que comprometen la integralidad de quien realiza el cuidado, las enfermeras construyen en su cotidianidad el saber relacionado con la presencia real e inevitable de los riesgos (Slovic, 1962), de tipo físico, químico, ergonómico, emocional, tejiendo desde su cotidianidad estrategias evidenciadas en la práctica sistematizada de las precauciones estándar de bioseguridad, superando la vulnerabilidad que como seres humanos las alcanza (Torralba \& Rosselló 1999; Cardona, 2010). Un aspecto vital consiste en el reconocimiento que a pesar de la existencia del riesgo percibido, prima la libertad humana para decidir por un comportamiento seguro o no; mostrarse invulnerable manifiesta la proximidad del accidente, por ello el comportamiento evidenciado en los testimonios de las enfermeras revela que en su sistema de creencias, valores, sentimientos y actitudes, conocen el riesgo e intentan protegerse (Slovic, 2000).

La permanencia y prolongación de la exposición al riesgo laboral se revela como expresión de la naturaleza misma del cuidado: ayudar a satisfacer necesidades específicas en un cuidado personalizado y a la par brindar la compañía, la ternura y comprensión que toda persona con déficit de salud anhela (Waldow, 1998), las heridas y molestias del cuerpo van por lo general acompañadas de heridas del alma dada nuestra unicidad y holismo; al cuidar al ser humano la enfermera ejerce una misión esencialmente relacional, vinculando a quienes cuida su pensar y sentir y no solo el hacer.

Los argumentos descritos permiten comprender la percepción vertida por las enfermeras del servicio de medicina y extraer la sabiduría que expresan sus diálogos: No sentirse igual que antes, reconocer que aparte de la carga física existe carga mental y emocional, percatarse de la posibilidad de ser agredidos y sufrir consecuencias legales por aspectos ligados al trabajo; son más que motivos suficientes para aceptar que aun con las mejores competencias personales y profesionales, el cuerpo se fatiga y el significado que se construye acerca del modo como este proceso se realiza, tiene enorme repercusiones para la Seguridad y Salud en el trabajo (Cifre, Salandava \& Franco 2011; OIT 2014).

Las implicancias de los riesgos laborales para la salud de la enfermera son una expresión del modo de compartir la existencia en una Sociedad de Riesgo (Beck 1998), donde el riesgo afirma su omnipresencia sin opción para elegir o no. Es relevante que mientras la demanda de cuidados se incrementa por conductas pasivas y acríticas respecto a la propia salud, se presente tantas dificultades a la hora de tomar decisiones políticas que propicien la transformación de las condiciones riesgosas que compete en gran medida a quien detenta el poder político como autoridad.

La categoría concerniente a la urgencia de superar los limitantes por una cultura preventiva, pone de relieve aspectos de suma trascendencia para la seguridad laboral y son en esencia condiciones de trabajo básicas que se encuentran de alguna manera soslayadas con incidencia desfavorable para la realización del cuidado.

Respecto a la subcategoría sobre la percepción innegable de trabajar con mobiliario y equipo obsoleto e insuficiente, no acorde a la naturaleza del cuidado de las personas es explicado por Santos y Mauro (2010), al enfatizar que cuanto más deterioro y carencia de espacio físico exista en la infraestructura, muy antigua en algunos establecimientos de salud, más disconfort y contracturas musculares dolorosas se presentarán en el personal; las lesiones de columna vertebral, el ausentismo y la insatisfacción laboral, revelan sin duda marcado déficit de gestión del riesgo laboral.

El patrón irregular de desabastecimiento de materiales de protección, el déficit de personal y la 
poca organización del trabajo, aunada a la falta de autonomía para manejar los recursos (Cárdenas, Arana, Monroy \& García 2010), hace más difícil una adecuada relación riesgo/confianza (Beck 1998).

El abordaje de la subcategoría del cuidado del cuidador, se evidencia una postura de desvaloración del cuidado, al impedir la existencia de condiciones para su ejecución óptima, que no se condice con los lineamientos de humanización declarados institucionalmente. Stein (2008), manifiesta que ante la responsabilidad de ejercer liderazgo en el marco de objetivos y metas organizativas comunes al equipo de salud, es importante construir de manera colectiva e integral las condiciones para el cuidado de las personas en el sistema de salud.

Urge por tanto, hacer consciencia de las consecuencias de los roles que desempeñan las enfermeras en condiciones deficitarias, exigiendo la evaluación de las condiciones actuales, su impacto en la salud $\mathrm{y}$ en la productividad, precisando un compromiso concreto con la mejora en la gestión de los recursos y equipamiento necesario, precisado en la Legislación vigente para el ejercicio eficiente de la enfermera como sujeto de derecho y respeto por el valor intrínseco de su vida en la organización.

La última y relevante categoría temática, se centra en el valor real del cuidado y de quien cuida en la percepción de las enfermeras sobre los riesgos laborales. Cabe señalar que la eficacia y eficiencia de los servicios de salud depende de la calidad de vida de quienes ejecutan los procesos de cuidado. En tal sentido invertir en mejores condiciones de trabajo, es apostar por la calidad del cuidado y el reconocimiento de seriedad en la prestación de servicios humanizados (Bermejo, 1999).

La canalización de soluciones a la problemática existente solo es posible desde una visión sistémica, porque es esta conciencia del riesgo que emerge en la percepción de las enfermeras, la condición que precede y preside las decisiones a tomar (Luhmann, 1998). La enfermera es un instrumento fundamental para el logro de los objetivos del milenio (OMS 2007); sin embargo para que el cuidado ocurra en la magnitud que se necesita, es necesario desarrollar consciencia del cuidado, esto engloba: discernimiento, intuición, pensamiento crítico y sensibilidad (Waldow, 2014).

Dar valor al cuidado es un desafío para autoridades y la propia enfermera; es salir de una postura acrítica y casi mimetizada con la situación existente para buscar puntos de encuentro comunes, recordando que existen en el centro del diálogo, derechos laborales irrenunciables, no sujetos a negociación.
Siendo las consideraciones finales, la percepción de los riesgos laborales en el proceso de cuidado de la enfermera en el contexto hospitalario, se revela de manera real y constante; el riesgo opera a modo de amenaza que se incrementa por la permanencia prolongada y la inseparabilidad de las dimensiones del cuidado instrumental y expresivo o sensible. Entre las categorías temáticas son evidentes los riesgos más conocidos y frecuentes: contraer enfermedades infecciosas y sufrir punciones accidentales; el riesgo por sobrecarga con evidentes molestias en la columna vertebral y a continuación, los riesgos de implicación emocional y estrés, ubicados como riesgos psicosociales.

Riesgos emergentes, son principalmente ser agredido por el propio paciente o la familia, quienes pueden haber sobrepasado los límites de la tolerancia al sufrimiento, ver amenazada su integridad o por malentendidos; constituye un elemento novedoso en el estudio y verse implicado en demandas legales, principalmente por caídas de paciente con consecuencias funestas o la decisión silenciosa de suicidio por parte del paciente.

La situación descrita, culmina en la urgencia de superar los limitantes por una cultura preventiva; se precisan la renovación de mobiliario y equipo considerado obsoleto, encauzar la ausencia del cuidado del cuidador y reparar en el escaso valor que se concede al cuidado y a quien cuida, aportaciones trascendentales en la tendencia internacional por parte de los organismos responsables de la salud laboral del trabajador, bajo la denominación de humanización del ambiente de trabajo.

Las condiciones de trabajo desfavorables para la enfermera, contrastan con las declaraciones de formar parte de los derechos amparados por ley y poseer por tanto, carácter exigible. Se infiere que sin un compromiso real para insertar en los planes institucionales, estrategias que viabilicen las políticas de seguridad y salud en el trabajo, no es posible mejorar la salud laboral ni las condiciones para realizar el cuidado, decisión equivalente a permanecer en una postura reduccionista y no humanizada respecto al trabajo.

Del mismo modo es indispensable materializar una revisión crítica de la postura de las enfermeras y superar la actitud de estar sobreviviendo en medio de un sistema que no contempla las necesidades básicas para brindar el cuidado en su naturaleza multidimensional y esencialmente relacional. Las implicancias para la institución, se orientan a asumir mejor el cuidado de la cuidadora (or) enfermera (o), 
Implicancias de los riesgos laborales percibidos por las enfermeras en el valor concedido al cuidado y al cuidador en el Servicio de Medicina Interna del Hospital Nacional Guillermo Almenara Irigoyen, Lima, 2016

ellas (os), son el 60\% de la fuerza de trabajo que labora en ámbito hospitalario y su derecho a la vida lo exige. Continuar sin una atención sistémica a esta situación equivale a perpetuar la inequidad de género por ser una profesión con contingente altamente femenino.

Es importante consignar a modo de reflexión, que siendo la salud laboral una responsabilidad compartida entre empleadores y el personal, es menester un mayor compromiso de la gestión y de los líderes involucrados para generar y llevar a la práctica planes y programas específicos que viabilicen las principales líneas de acción a implementar en el mediano plazo: abastecimiento regular de insumos de protección, estudios sobre productos para limpieza menos nocivos para la vía respiratoria, plan de adquisición de sillas, grúas y camillas de altura graduable que aseguren el manejo ergonómico libre de riesgo en las actividades de mayor frecuencia en el cuidado. Se impone, actualizar y sincerar el Plan anual de dotación de enfermeras y técnicos de enfermería, concordante con el grado de dependencia/riesgo y la calidad del cuidado que amerita el paciente hospitalizado.

\section{Declaración de financiamiento y de conflicto de intereses:}

El estudio fue financiado por los autores, quienes declaran no tener algún tipo de conflicto de interés en la investigación realizada.

\section{Correspondencia:}

Irene Zapata Silva

Oficina Gestión de la Calidad. Hospital Nacional Guillermo Almenara Irigoyen. Av. Grau 800, La Victoria, Lima Perú.

e-mail: delicirenezapatas@yahoo.es.

\section{REFERENCIAS BIBLIOGRÁFICAS}

Bermejo JC. (1999) Salir de la Noche. Barcelona: Ed. Salterrae.

Cárdenas, Arana, Monroy y García. (2010). Cuidado profesional de enfermería. México D.F.: Federación mexicana de Asociaciones y Facultades de enfermería.

Cardona. (2010). La necesidad de repensar de manera holística los conceptos de vulnerabilidad y riesgo. Bogotá: Universidad de los Andes.

Cifre F. (2011). Riesgos psicosociales de hombres y mujeres. Revista Gestión Práctica de riesgos laborales. Universidad Jaume de Castellón Feb.; vol. 2 (82): 2526.

Creus A. (2013). Técnicas para la Prevención de Riesgos Laborales. Barcelona: Técnicas Mancorbo.

Firth A. Etnometodología. Discurso y Sociedad. (2010). Universidad de New Castle; 4(3). Disponible en: http:// www.dissoc.org.

Luhmann N. (1998). Sociología del riesgo. Berlín: Triana.

Meneguini F, Paz A, Lautert L. Factores ocupacionales asociados a los componentes del Síndrome Burn out en trabajadores de enfermería. Rev. Texto y Contexto, abr/ jun.; 20(2): 225-33.

Ministerio de Salud (2013). Segunda Medición de Metas Regionales de Recursos Humanos para la Salud. Perú 2007-2015. Lima: Dirección General de Recursos Humanos. Serie Bibliográfica Recursos Humanos en Salud $N^{\circ} 15$. Disponible en: http://www.minsa.gob.pe/ dggdrh/libros/pdf/s1/I-15.\%20segunda\%20Medición.

Murofuse N, Abranches S, Napoleao A. (2005). Reflexiones sobre el estrés y el Burn out y sus relaciones en enfermería. Rev. Lat. De Enf. s/m; 13(2): 255-61.
Organización Internacional del Trabajo. (2014). Aportes para una cultura de prevención. Buenos Aires: Ministerio de Trabajo, empleo y Seguridad social.

Organización Panamericana de la Salud/OMS. (2015). La Política de recursos humanos y el compromiso con el derecho a la salud. Resultados de la Primera medición de metas regionales de Recursos Humanos 2007-2015 en la Región andina. Disponible en: http:// www. observatoriorh.org.

Santos N, Mauro MY. (2009). Análisis de los factores de riesgo ocupacionales del trabajo de enfermería, desde la óptica de las enfermeras. Rio de Janeiro. Rev bras. Saúde ocup s/m. Universidad de Río de Janeiro; 35(121): 157-167. Disponible en: http://www.scielo.br/ pdf/rbsolv35n121/17.pdf.

Slovic P. (2000). Percepción del riesgo. Earthscan, London: Earthscan.

Stein D, Stein M, Macedo F, Lorenzini A. (2008). El rol del enfermero en el contexto hospitalario: visión de los profesionales de la salud. Cienc Cuid Saúde; 7 (3): 319-26.

Waldow VR. (1998). Cuidado humano: el rescate necesario. Petrópolis-Brasil: Vozes.

Waldow VR. (2014). Cuidado de Enfermería. Reflexiones entre dos orillas. España: Coord. Editorial Manuel Amezcua.

Recibido: 15/03/2016 Aceptado: 02/05/2016 